\title{
Highly efficient lithium utilization in lithium metal full-cell by simulated missile guidance and confinement systems
}

\author{
Jinqiu Zhou ${ }^{1 \dagger}$, Haoliang $\mathrm{Lu}^{1 \dagger}$, Xi Zhou ${ }^{2 *}$, Haoqing Ji ${ }^{1}$, Yawen Sun ${ }^{1}$, Tao Qian ${ }^{1^{*}}$ and \\ Chenglin $\operatorname{Yan}^{1 *}$
}

\begin{abstract}
The efficient utilization of metallic lithium (Li) is the key to enable application of $\mathrm{Li}$ metal full-cell with low amount of excess $\mathrm{Li}$, contributing to higher safety and energy density. Herein, we report an extraordinary $\mathrm{Li}$ metal full-cell with only $20 \%$ excess $\mathrm{Li}$, which demonstrated significantly improved reversibility and high Coulombic efficiency. Ingenious simulated missile guidance and confinement system (SMGCS) was designed to guide and confine $\mathrm{Li}$ deposition through constructing compatible silver lithiophilic sites and nitrate layer. Silver sites act as effective $\mathrm{Li}$ nuclei to attract $\mathrm{Li}$ ions and direct the initial nucleation. The generated nitrate layer affords an interfacial environment favorable for confined and uniform deep Li deposition, which is theoretically verified by molecular dynamics (MD) simulations. The two combined merits offer a robust and dendrite-free $\mathrm{Li}$ deposition, enabling the application of $\mathrm{Li}$ metal full-cell with slight excess $\mathrm{Li}$. They also result in an outperformed $\mathrm{Li}$ cycling efficiency of ca. $99 \%$ for over 300 cycles along with deep cycling at a high capacity of $10 \mathrm{~mA} \mathrm{~h} \mathrm{~cm}{ }^{-2}$ in carbonate electrolytes. The unprecedented high degree of $\mathrm{Li}$ utilization opens a new avenue for the future development of highly efficient $\mathrm{Li}$ metal full-cells.
\end{abstract}

Keywords: lithium utilization, lithium metal, full-cell, Li nucleation, Li deposition

\section{INTRODUCTION}

Metallic Li, the ultimate anode for Li-based and perhaps all next-generation rechargeable batteries with the highest specific capacity $\left(3860 \mathrm{~mA} \mathrm{~h} \mathrm{~g}^{-1}\right)$ and lowest redox potential $(-3.040 \mathrm{~V} v s$. the standard hydrogen electrode) of all possible alternatives [1-3], is encountering significant obstacles: the low degree of Li utilization, poor reversibility, and dendrite formation during cycling, which is responsible for low Coulombic efficiency (CE), large voltage polarization, poor capacity retention and shortcircuiting [4-7]. The major issue of fast Li loss exists during Li metal full-cell operation, thus more than $200 \%$ excess $\mathrm{Li}$ is demanded to avoid capacity degradation. Moreover, excess Li exacerbates safety problems and decreases the energy density [8]. These issues hinder the large-scale applications of Li metal anodes. Therefore, the challenge towards the practical applications of $\mathrm{Li}$ metal batteries (LMBs) is to achieve excellent stability with $\mathrm{Li}$ loading as low as possible (i.e., higher degree of Li utilization) to obtain higher safety level and energy density. It has been known that ether-based electrolytes have significant impact on the uniform deposition of $\mathrm{Li}$, offering high CE [9]. However, the high flammability and low oxidation decomposition potential $\left(<4 \mathrm{~V} v s . \mathrm{Li}^{+} / \mathrm{Li}\right)$ of ether solvents does not satisfy the required safety indexes in widely-used high-voltage cathodes, hindering their widespread application [9]. High-salt-concentration ether electrolytes have been recently proposed, but their economic effectiveness need to be evaluated for large-scale commercial applications [9-12]. Conversely, low-cost carbonate-based electrolytes such as $1 \mathrm{~mol} \mathrm{~L}^{-1} \mathrm{LiPF}_{6}$ in ethylene carbonate (EC)/diethyl carbonate (DEC) (1:1 volumetric ratio) exhibit lower flammability and higher voltage tolerance; thus, they could be applied in almost all commercial Li-ion batteries (LIBs). Nevertheless, carbonate electrolytes are highly corrosive to Li metal, resulting

\footnotetext{
${ }^{1}$ Soochow Institute for Energy and Materials Innovations, College of Energy, Key Laboratory of Advanced Carbon Materials and Wearable Energy Technologies of Jiangsu Province, Soochow University, Suzhou 215006, China

${ }^{2}$ Institute of Chemical Industry of Forestry Products, Chinese Academy of Forestry, Nanjing 210042, China

These two authors contributed equally to this work.

* Corresponding authors (emails: zhouxi1123nju@foxmail.com (Zhou X); tqian@suda.edu.cn (Qian T); c.yan@suda.edu.cn (Yan C))
} 
in catastrophic growth of $\mathrm{Li}$ dendrite and poor cycling efficiency $[9,13]$. Therefore, these severe problems for LMBs with carbonate-based electrolytes urgently need to be solved. Extensive efforts have been devoted to improving the efficiency and cycle life of Li metal electrodes in carbonate-based electrolytes $[9,13-17]$, such as carefully designed solid-electrolyte interfaces (SEI) based on different additives including nitrogenous and fluorinated compounds [13-15], protective coatings [16-18], nanostructured electrodes [19-21], and separator modification [22]. However, so far, the cycling efficiency in carbonate-based electrolytes is barely satisfactory accompanied by a low degree of Li utilization. In the present work, our target is to achieve excellent cycling efficiency at high capacity in commonly employed carbonate-based liquid electrolytes and to realize high $\mathrm{Li}$ utilization in Li metal full-cells.

It is well-known that the initial nucleating behavior of Li could largely determine the final deposition morphology. Substrates with 'lithiophilic' sites with great affinity of metallic $\mathrm{Li}$ could reduce $\mathrm{Li}$ nucleation barrier. Therefore, it is highly desirable to introduce lithiophilic nucleation sites, which could serve as nucleation seeds towards directing Li nucleation and growth at the initial process of Li plating. Nearly zero nucleation overpotential is needed to nucleate $\mathrm{Li}$ in some types of metals, including $\mathrm{Au}$ and Ag, which exhibit a specific Li solubility [21]. Furthermore, during the long-term and deep cycling, an interfacial environment favorable for attracting $\mathrm{Li}$ ions towards a homogeneous $\mathrm{Li}$ ion flux distribution and uniform Li deposition is of crucial importance for electrochemical cycling of metallic $\mathrm{Li} . \mathrm{LiNO}_{3}$ is usually used for SEI formation in ethers for Li-sulfur batteries due to the preferential reduction of nitrate anions $\left(\mathrm{NO}_{3}{ }^{-}\right)$instead of other electrolyte components. In particular, $\mathrm{LiNO}_{3}$ could substantially alter the interfacial chemistry via the formation of nitrogen-containing species, such as $\mathrm{Li}_{3} \mathrm{~N}$ and lithium oxynitrides $\left(\operatorname{LiN}_{x} \mathrm{O}_{y}\right)$, which have been long considered to possess the highest ionic conductivity rendering dendrite-free, reversible, and highly dense metallic $\mathrm{Li}$ deposition [9,23-25]. However, $\mathrm{LiNO}_{3}$ remains less explored in carbonate-based electrolytes due to its severe solubility limitation thereby leading to unsustainable decomposition and weak SEI.

Herein, we simulated missile guidance and confinement systems (SMGCS) to realize pronounced stability and unprecedentedly high degree of Li utilization (merely $20 \%$ excess anodic capacity in Li metal full-cell) by simultaneously inducing and confining Li deposition from the perspective of both initial nucleation stage and the following deep-cycling stage. Silver lithiophilic sites and an in-situ formed nitrate layer are achieved on the surface of Li metal anode through a facile displacement reaction between the deposited $\mathrm{Li}$ and introduced $\mathrm{AgNO}_{3}$ membranes due to the large electronegativity difference between alkali metals and metallic Ag [26-28]. The lithiophilic Ag could play the role of nucleation sites to reduce $\mathrm{Li}$ nucleation overpotential and guide the Li-ions in the initial nucleation process. Furthermore, the nitraterich layer provides favorable interfacial environment with narrow and smooth ionic transportation channels to confine and homogenize Li deposition, which was further verified by the MD simulations. The missile guidance and confinement systems synergistically act to enable uniform Li deposition with high reversibility in commonly used carbonate-based electrolytes without compromising the ionic conductivity, viscosity, and cost of the electrolytes. Moreover, an extraordinarily high utilization of Li metal anode can be achieved (merely $20 \%$ excess $\mathrm{Li}$, i.e., $\mathrm{Li}$ utilization of $83.3 \%$ ), which is expected to favor the safe use of $\mathrm{Li}$ metal anode, opening a new avenue for the future development of Li metal full-cells.

\section{EXPERIMENTAL SECTION}

\section{Preparation of the SMGCS}

$\mathrm{AgNO}_{3}$ was purchased from Alfa Aesar and mechanically ball-milled for five hours. The $\mathrm{Cu}$ substrates were cleaned by ethanol and water before use. Pre-calculated amount of $\mathrm{AgNO}_{3}$ powder was homogeneously dispersed in 1,2dimethoxyethane via ultrasonication by ultrasonic cell grinder. Droplet-coating technique was employed in the fabrication to prepare $\mathrm{AgNO}_{3}$ membrane on $\mathrm{Cu}$ under darkness at room temperature. After slow evaporation of the solvent, the substrate was rolled by electric rolling machine.

\section{Material characterization}

The in-situ X-ray diffraction (XRD) measurements were performed on the X-ray diffractometer (D8 ADVANCE, Bruker AXS GmbH Co., Ltd). Argon ion etching was conducted on an X-ray photoelectron spectroscope (XPS) in an ultra-high vacuum ESCALAB 250 set-up equipped with a monochromatic $\mathrm{Al} \mathrm{Ka} \mathrm{X}$-ray source $(1486.6 \mathrm{eV}$; anode operating at $15 \mathrm{kV}$ and $20 \mathrm{~mA}$ ). The in-situ observation for the Li deposition was performed on a metallurgical microscope (Caikon Optical Instrument DMM-330C). Morphologies were observed by scanning electron microscopy (SEM, SU8010, Japan) and transmission electron microscopy (TEM, Titan Themis Cubed 
G2 300).

\section{Electrochemical characterization}

A commercial electrolyte based on $1 \mathrm{~mol} \mathrm{~L}^{-1} \mathrm{LiPF}_{6}$ in EC/ DEC (1:1 volumetric ratio) was used. The assembly of the $\mathrm{Li} \| \mathrm{Cu}$ cell was conducted in an Ar-filled glovebox. The amount of electrolyte used in the $\mathrm{Li} \| \mathrm{Cu}$ cells was $20 \mu \mathrm{L}$. The CE of a cell was defined by dividing the stripped capacity to the plated capacity at each charging-discharging cycle. Tests with excess Li were also performed: Li with a certain amount was plated (in accordance with the amount of excess $\mathrm{Li}$ ), followed by some Li stripping/ plating cycles. Finally, the cell was charged to $1 \mathrm{~V}$. Then we could obtain the average $\mathrm{CE}$ as the ratio of the total stripped Li to that of the plated Li. The long term stability of Li metal with a certain amount of excess $\mathrm{Li}$ was also conducted. Cu or SMGCS electrodes were pre-deposited with certain amounts of $\mathrm{Li}$ as anodes and then they were paired with cathodes to constitute Li metal full-cells. The "lithium utilization" is the ratio of cathode capacity to anode capacity in the lithium metal full-cell. The mass loading of $\mathrm{LiCoO}_{2}$ (LCO) cathode (purchased from company of HF-kejing) was about $18.5 \mathrm{mg} \mathrm{cm}^{-2}$. The amount of electrolyte used in the Li||LCO cells was $50 \mu \mathrm{L}$ and the electrolyte-to-capacity ratio was $\sim 18 \mu \mathrm{L} \mathrm{mA}^{-1} \mathrm{~h}^{-1}$. The current density of the Li||LCO was $2 \mathrm{~mA} \mathrm{~cm}^{-2}$, and the voltage range was $4.45-3 \mathrm{~V}$ vs. $\mathrm{Li} / \mathrm{Li}^{+}$. The voltage range of $\mathrm{Li}|| \mathrm{LiNi}_{1 / 3} \mathrm{Co}_{1 / 3} \mathrm{Mn}_{1 / 3} \mathrm{O}_{2}(\mathrm{NCM})$ was $4.3-2.7 \mathrm{~V}$ vs. $\mathrm{Li} / \mathrm{Li}^{+}$. The battery performances were tested by using a battery testing system (LAND CT 2001A, Wuhan, China). The EIS measurements were conducted on a $\mathrm{CHI}$ 660E (Chenhua Shanghai, China) electrochemical workstation.

\section{MD simulations}

MD simulations were carried out with the open source software package Gromacs, version 5.0.5. The initial configurations were constructed by dispersing certain amounts of $\mathrm{LiNO}_{3}$ molecules $(0,16,1600)$ into the interface environments $\left(480 \mathrm{EC}+256 \mathrm{DEC}+64 \mathrm{LiPF}_{6}\right.$ molecules). EC and DEC were simulated by the OPLS-AA force field. $\mathrm{NO}_{3}{ }^{-}$and $\mathrm{PF}_{6}{ }^{-}$ions were parameterized according to the literatures $[29,30]$. For $\mathrm{Li}^{+}$ions in $\mathrm{LiNO}_{3}$ and $\mathrm{LiPF}_{6}$, the parameters were obtained from a document [31]. Intermolecular van der Waals interactions were represented by the Lennard Jones (LJ) potential with the geometric combination rules. LJ interactions as well as the short-range electrostatic interactions were truncated at $1.2 \mathrm{~nm}$, while the long-range electrostatic interactions were calculated by the particle mesh Ewald algorithm with a Fourier spacing of $0.12 \mathrm{~nm}$ in the reciprocal space. For all simulation systems, energy minimization was first performed with the steepest descent algorithm, followed by isothermal-isobaric equilibration at $298.15 \mathrm{~K} / 1$ bar for $50 \mathrm{ps}$. Then, production runs were carried out under NPT ensemble for $20 \mathrm{~ns}$, with system temperature and pressure controlled by the Nose-Hoover thermostat and Parrinello-Rahman barostat, respectively. The periodic boundary conditions were applied in all directions. Leap-frog algorithm with a time-step of $1 \mathrm{fs}$ was used to integrate the motion equations during simulations.

\section{RESULTS AND DISCUSSION}

According to the Li-Ag phase diagram (Fig. S1), Ag has an appreciable solubility in $\mathrm{Li}$ and can generate a solid solution buffer layer before Li metal formation. Therefore, it could improve the lithiophilic nature and lower the deposition interface energy between current collectors and Li metal. As schematically illustrated in Fig. S2a, when lithiophobic $\mathrm{Cu}$ was employed as the substrate, the nucleation overpotential of $\mathrm{Li}$ metal onto the pristine $\mathrm{Cu}$ is much higher and the isolated initial nucleation sites are randomly distributed on the heterogeneous and rough $\mathrm{Cu}$ surface, which leads to random Li deposition underneath [32]. Consequently, the surface of deposited Li metal is not uniform and the large number of protuberances would enhance the local electric field and attract Li ions, leading to a dendritic morphology [33]. Thereby, we need to introduce lithiophilic nucleation sites serving as nucleation seeds to attract and guide Li ions due to their low surface diffusion energy barrier [19]. Nearly zero nucleation overpotential is needed to nucleate Li metal on $\mathrm{Ag}$, which could act as an effective Li nuclei, facilitating $\mathrm{Li}$ nucleation and growth (Fig. S2b). However, upon continuous Li plating, the nucleation sites are progressively covered by the Li layer. Thus, it is of particular importance to synergistically combine the lithiophilic nucleation sites with an efficient ionic conductive interface film together, which is beneficial for a homogeneous $\mathrm{Li}$ ion flux distribution. As a result, we proceed to SMGCS novel system to combine the lithiophilic Ag nucleation sites with $\mathrm{LiNO}_{3}$-rich layer through the facile displacement reaction between the deposited $\mathrm{Li}$ and introduced $\mathrm{AgNO}_{3}$ membrane on the current collector. This specific approach can lead to uniform Li nucleation and dendritefree growth during the initial nucleation and subsequent plating process (Fig. S2c). The SEM and energy dispersive spectrometer (EDS) characterizations for $\mathrm{AgNO}_{3}$ layer on the $\mathrm{Cu}$ substrate were conducted (Figs S3 and S4). Ob- 
viously, the $\mathrm{AgNO}_{3}$ layer was smoothly and homogeneously distributed on the substrate, which is helpful for the uniform $\mathrm{Li}$ deposition. The influences of the thickness and roughness of $\mathrm{AgNO}_{3}$ layer on battery performances were also estimated. Clearly, the thickness of $200 \mathrm{~nm}$ was enough for the excellent battery performance (Fig. S5). More $\mathrm{AgNO}_{3}$ would worsen the energy density of batteries. Smoothly and homogeneously distributed $\mathrm{AgNO}_{3}$ layer on the substrate was helpful for the uniform $\mathrm{Li}$ deposition (Fig. S6).

MD simulations were conducted to explore the different interfacial environments in different $\mathrm{Li}$ /electrolyte systems. Schematic for interfacial environments of ordinary interface in pristine EC/EDC (Fig. 1a), interface with little $\mathrm{LiNO}_{3}$ (Fig. 1b), $\mathrm{LiNO}_{3}$-rich layer (Fig. 1c) and their corresponding MD simulation boxes (Fig. $1 \mathrm{~d}-\mathrm{f}$ ) are illustrated. Distinctly, the molecules EC/DEC are the major components for the ordinary interface. According to the integral radial distribution functions (RDF) for $\mathrm{Li}-\mathrm{O}_{\mathrm{EC} / \mathrm{DEC}}$ in Fig. 1g, four oxygen atoms from the electrolyte solvents EC/DEC can randomly interact with a $\mathrm{Li}$ ion in the ordinary interface. In addition, the introduction of a low amount of $\mathrm{LiNO}_{3}$ in the interfacial environment can decrease the $\mathrm{Li}-\mathrm{O}_{\mathrm{EC} / \mathrm{DEC}}$ number, indicating less interaction between $\mathrm{O}_{\mathrm{EC} / \mathrm{DEC}}$ and $\mathrm{Li}$ ions (Fig. 1g). The appearance of new $\mathrm{Li}-\mathrm{O}_{\mathrm{NO}_{3}}{ }^{-}$entities (Fig. 1h) also proves the participation of nitrate traces in the interaction between Li ions. More importantly, the $\mathrm{LiNO}_{3}$-rich layer exhibits a profound impact on the $\mathrm{Li}$ surface chemistry. A sharp drop of $\mathrm{Li}-\mathrm{O}_{\mathrm{EC} / \mathrm{DEC}}$ (Fig. 1g) along with a sharp increase of $\mathrm{Li}-\mathrm{O}_{\mathrm{NO}_{3}}{ }^{-}$number is taking place and about 3.5 oxygen atoms from $\mathrm{NO}_{3}{ }^{-}$interact
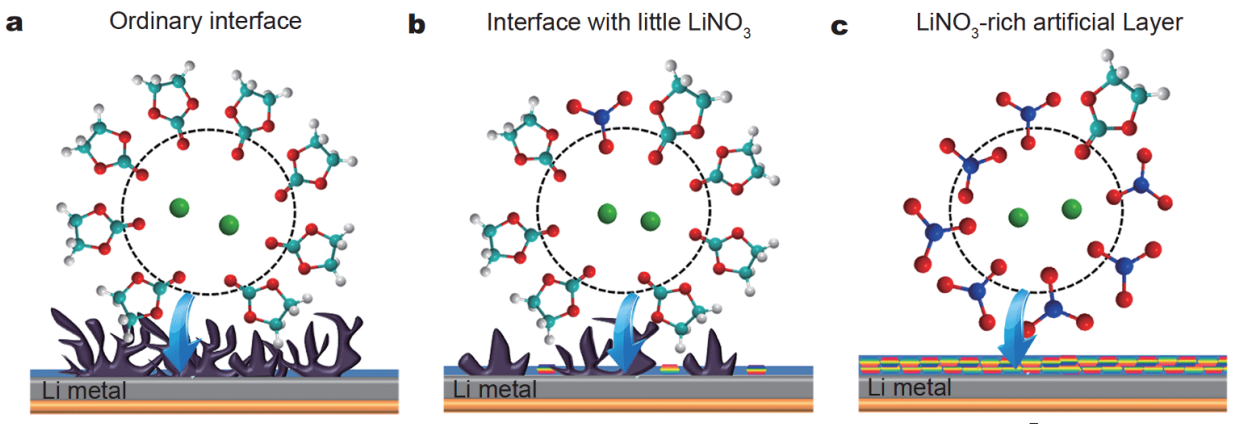

d

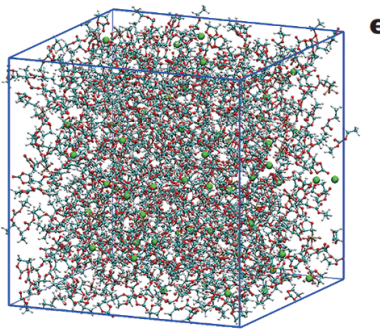

西
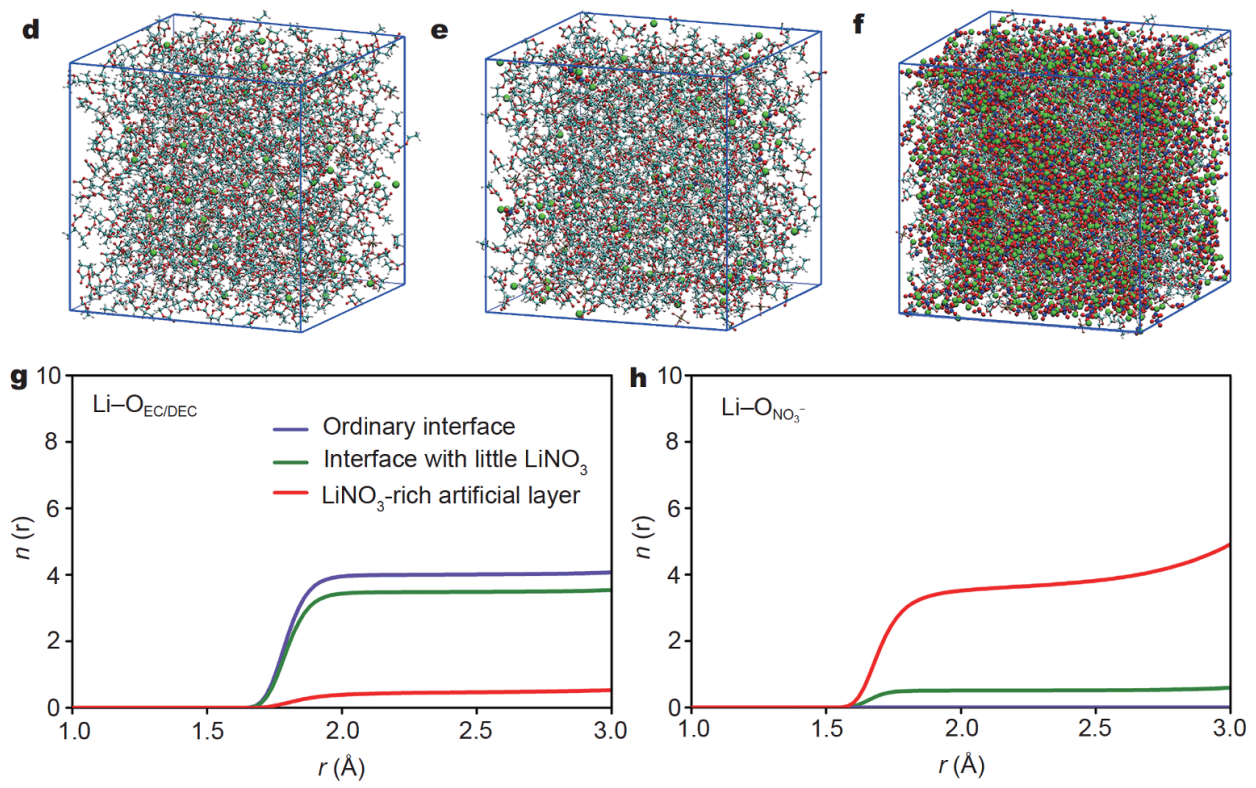

Figure 1 Illustrations and MD simulation results for different interfacial environments. Schematic illustrations and MD simulation boxes of (a, d) ordinary interface in pristine EC/EDC, (b, e) interface with little $\mathrm{LiNO}_{3},(\mathrm{c}, \mathrm{f}) \mathrm{LiNO}_{3}$-rich layer. Li: green; O: red; C: teal; $\mathrm{H}$ : grey; $\mathrm{N}$ : blue; F: pink; P: tan. (g) Integral $\mathrm{RDF}$ for $\mathrm{Li}-\mathrm{O}_{\mathrm{EC} / \mathrm{DEC}}$. (h) Integral $\mathrm{RDF}$ for $\mathrm{Li}-\mathrm{O}_{\mathrm{NO}_{3}}{ }^{-}$. 
with $\mathrm{Li}$ ion (Fig. 1h). The strong interaction of $\mathrm{NO}_{3}^{-}$with $\mathrm{Li}$ ions within the $\mathrm{LiNO}_{3}$-rich layer provides narrow and smooth ionic transportation channels to induce and confine Li-ion flux, which is just like that a missile is precisely guided and confined to the destination controlled by MGCS. Besides, the favorable interfacial environment in the $\mathrm{LiNO}_{3}$-rich layer results in a small probability of EC/DEC decomposition, which would be beneficial for the avoidance of the formation of fragile and unstable SEI.

To further unveil the origin of superiority of the designed SMGCS Li metal anode, in-situ galvanostatic cycling XRD characterization was used to monitor the $\mathrm{Li}$ plating behavior and to estimate the phase composition and changes in real time during the plating process (Fig. 2a). The initial XRD patterns demonstrate characteristic diffraction peaks of the starting phase $\left(\mathrm{AgNO}_{3}\right)$ at $21.50^{\circ}, 24.35^{\circ}, 29.72^{\circ}, 31.97^{\circ}, 32.86^{\circ}, 35.58^{\circ}, 38.99^{\circ}$, $39.18^{\circ}, 40.18^{\circ}, 41.73^{\circ}, 42.78^{\circ}$ and $43.55^{\circ}$. Along with slow $\mathrm{Li}$ deposition, it is clear that these peaks for $\mathrm{AgNO}_{3}$ progressively weaken and finally vanish. Then two new featured peaks of metallic Ag appear at $38.23^{\circ}$ and $44.3^{\circ}$, and peaks for $\mathrm{LiNO}_{3}$ could also be observed at $32.1^{\circ}, 35.4^{\circ}$ and $42.5^{\circ}$, indicating the gradual consumption of $\mathrm{AgNO}_{3}$ and the generation of $\mathrm{Ag}$ and $\mathrm{LiNO}_{3}$ [34]. Furthermore, XPS analysis was carried out on electrodeposited Li nuclei with step-by-step sputtering to exactly analyze the depthdependent chemical composition distribution of the $\mathrm{AgNO}_{3}$-derived interface film. Fig. $2 \mathrm{~b}$ depicts the depthdependent Ag $3 \mathrm{~d}$ and $\mathrm{N}$ 1s XPS spectra. The peaks at 367.5 and $373.5 \mathrm{eV}$ on the surface are assigned to $\mathrm{Ag} 3 \mathrm{~d}_{5 / 2}$ and $\mathrm{Ag} \mathrm{3d_{3/2 }}$ of $\mathrm{Ag}^{+}$, respectively, in the original $\mathrm{AgNO}_{3}$. The peaks shift upwards to 368.5 and $374.5 \mathrm{eV}$ indexed the featured peaks of $\mathrm{Ag}$ and the peak intensities increase with the sputtering thickness, indicating the formation of Ag lithiophilic nucleation sites at the bottom. In addition, the surface nitrogen species exhibit higher oxidation states $\left(\mathrm{LiNO}_{3}\right.$ and $\left.\mathrm{LiNO}_{2}\right)$, and $\mathrm{Li}_{3} \mathrm{~N}$ and $\mathrm{LiN}_{x} \mathrm{O}_{y}$ can be detected downward. The abundant $\mathrm{NO}_{3}^{-}$reveals a profound influence on the Li surface chemistry, towards the formation of $\mathrm{Li}_{3} \mathrm{~N}$ and $\mathrm{LiN}_{x} \mathrm{O}_{y}$ species via the preferential reduction of $\mathrm{NO}_{3}{ }^{-}$. These species have long been acknowledged to possess superior Li-ion conductivity and low Li-ion transfer resistance. Consequently, it is appar-
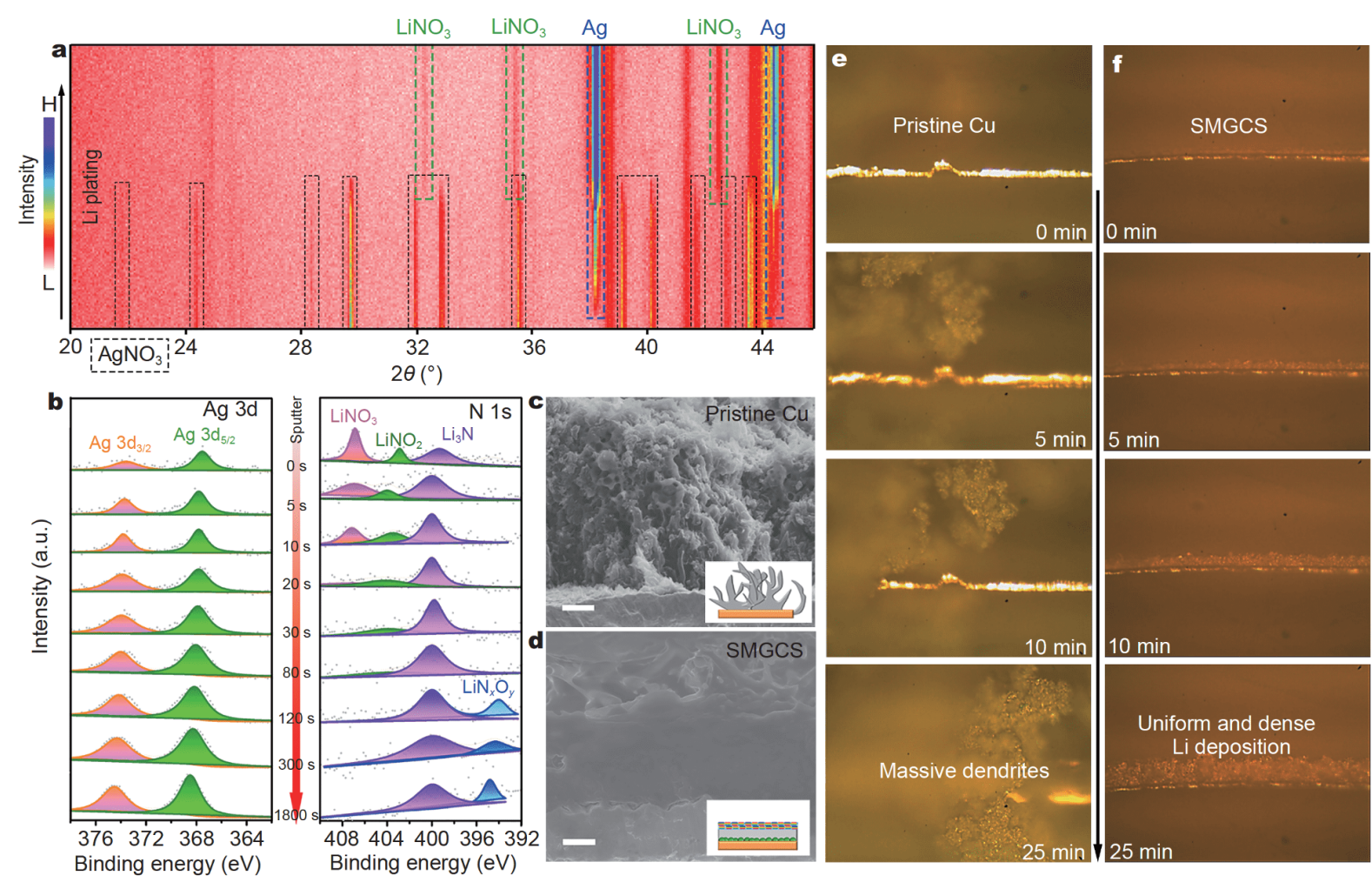

Figure 2 Investigations for the origin of superiority of the SMGCS. (a) In-situ galvanostatic cycling XRD characterization during initial Li plating process. (b) Depth-dependent Ag 3d and N 1s XPS spectra on the electrodeposited Li nuclei. Cross-sectional SEM images of the Li layer plated on the $\mathrm{Cu}$ current collector in the 20th cycle under $2 \mathrm{~mA} \mathrm{~cm}^{-2} \& 2 \mathrm{~mA} \mathrm{~h} \mathrm{~cm}^{-2}$ for Li\|Cu cells: (c) pristine Cu, and (d) SMGCS. The scale bar is $6 \mu \mathrm{m}$. In-situ optical microscopy for the deposition of Li for (e) pristine Cu foil and (f) SMGCS. 
ent that the introduction of $\mathrm{AgNO}_{3}$ membrane enables the SMGCS via the construction of compatible silver lithiophilic sites and nitrate layer. Ag sites with almost no nucleation barrier could act as effective Li nuclei to attract $\mathrm{Li}$ ions and direct the initial nucleation. On the other hand, the generated nitrate-rich layer can provide a favorable interfacial environment, which offers the required ionic transportation channels to induce and confine $\mathrm{Li}$ deposition. These particular SMGCS features synergistically contribute to an outperformed Li cycling efficiency and stability in commercial carbonate electrolytes. Intuitive visual perception was also acquired to verify its outstanding performance. SEM and TEM were conducted to monitor the morphology of Li deposits after 20th cycle (Fig. 2c, d and Figs S7 and S8). The Li deposits on pristine $\mathrm{Cu}$ electrode are dendritic and mossy with a loosely packed structure (Fig. $2 \mathrm{c}$ and Fig. S7). In stark contrast, a smooth, uniform and sturdy morphology without any traces of dendrites is displayed for the SMGCS Li deposits and the interface structure remains stable upon the increase of Li loading (Fig. 2d and Fig. S8). Furthermore, in-situ optical microscopy was conducted to intuitively observe the depositing behavior of Li. Dendrites grow fast and the pristine $\mathrm{Cu}$ foil is almost totally covered (Fig. 2e). In complete contrast, dense and uniform Li plating for SMGCS could be apparently observed (Fig. 2f), confirming the key role of SMGCS in controlling the dendrite-free morphology.

$\mathrm{Li} \| \mathrm{Cu}$ cells with a commercial carbonate electrolyte, $1 \mathrm{~mol} \mathrm{~L}^{-1} \mathrm{LiPF}_{6}$ in EC/DEC (1:1 volumetric ratio), were assembled to investigate the electrochemical Li plating/ stripping processes. The nucleation overpotential at the beginning of deposition, defined as the difference between the bottom of the voltage dip and the flat part of the voltage plateau [21,32], was employed to quantitatively assess the resistance of Li plating at the electrode surface (Fig. 3a). Regarding pristine $\mathrm{Cu}$, high overpotential of $\sim 62 \mathrm{mV}$ is needed to overcome the hetero-
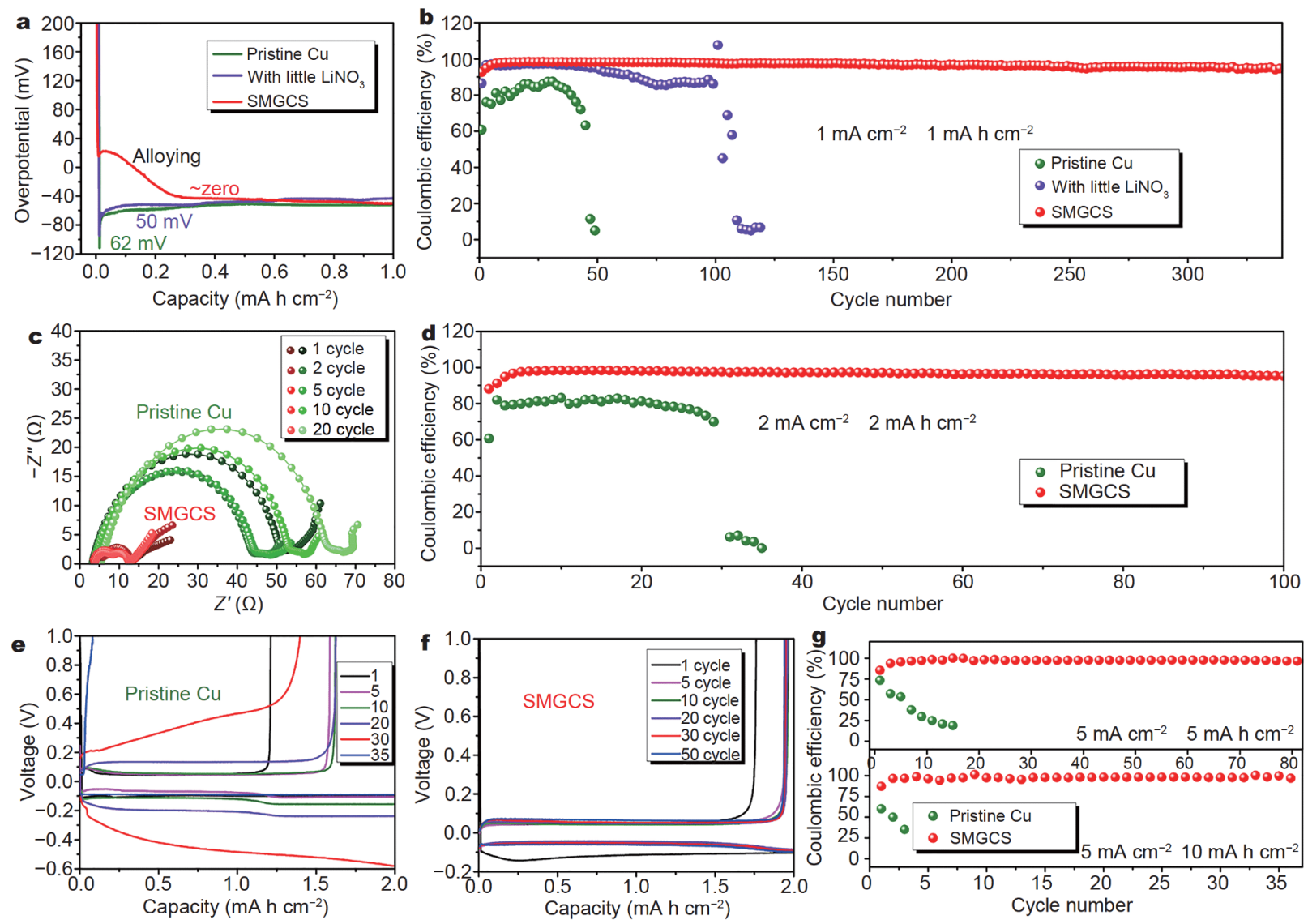

Figure 3 Electrochemical performance for Li\|Cu cells. (a) The nucleation overpotentials for Li metal. (b) CEs of Li $\| \mathrm{Cu}$ cells cycled under $1 \mathrm{~mA} \mathrm{~cm}^{-2}$ $\& 1 \mathrm{~mA} \mathrm{~h} \mathrm{~cm}^{-2}$. (c) The corresponding EIS spectra taken at the full Li-plated state $\left(1 \mathrm{~mA} \mathrm{~h} \mathrm{~cm}^{-2}\right.$ of Li plated on the Cu foil) after various numbers of cycles. (d) CEs of Li $\| \mathrm{Cu}$ cells cycled under $2 \mathrm{~mA} \mathrm{~cm}^{-2} \& 2 \mathrm{~mA} \mathrm{~h} \mathrm{~cm}^{-2}$. (e, $\mathrm{f}$ ) The corresponding discharge-charge curves of the Li\|Cu in (d). (g) CEs of $\mathrm{Li} \| \mathrm{Cu}$ cells cycled under $5 \mathrm{~mA} \mathrm{~cm}^{-2} \& 5 \mathrm{~mA} \mathrm{~h} \mathrm{~cm}^{-2}$ and $5 \mathrm{~mA} \mathrm{~cm}^{-2} \& 10 \mathrm{~mA} \mathrm{~h} \mathrm{~cm}^{-2}$. 
geneous nucleation barrier, due to the large thermodynamic mismatch between $\mathrm{Li}$ and $\mathrm{Cu}$. In addition, a relatively high overpotential of $\sim 50 \mathrm{mV}$ with little $\mathrm{LiNO}_{3}$ is also observed in the initial stage of Li nucleation, indicating the significance of nucleation seeds. Significantly, almost zero nucleation potential at the onset is observed for SMGCS. It can be attributed to the fact that almost no nucleation barrier exists for Li metal nucleating on $\mathrm{Ag}$ [21]. The CE of the cell, defined as the ratio of the amount of the stripped $\mathrm{Li}$ to that of the plated $\mathrm{Li}$ at each charging-discharging cycle, is frequently adopted as a performance index to evaluate the cyclability of the $\mathrm{Li}$ metal electrode. At the current density of $1 \mathrm{~mA} \mathrm{~cm}{ }^{-2}$ with capacity depth of $1 \mathrm{~mA} \mathrm{~h} \mathrm{~cm}^{-2}$, the cell of pristine $\mathrm{Cu}$ exhibits remarkably inferior cycling performance with an average CE of $80 \%$ only for 50 cycles. The CE performance for the cell with little $\mathrm{LiNO}_{3}$ is slightly improved. In complete contrast, the cell of SMGCS can be stably cycled for over 300 cycles, delivering a high CE of $~ 99 \%$ (Fig. 3b). Li ion transfer resistances for the Li\|C Cu cells upon cycling were also investigated to uncover the kinetic features of the electrode interfacial film (Fig. $3 \mathrm{c}$ and Fig. S9). The Nyquist plots of the cells of pristine $\mathrm{Cu}$ and with little $\mathrm{LiNO}_{3}$ additive exhibit large and changed interfacial resistance (including SEI layer resistance and charge transfer resistance) due to the crack and repair of fragile SEI film, which aggravates the deterioration for the battery performances. In contrast, the cell of SMGCS shows exceptionally small and stable resistance, indicating fast charge transfer rate, low diffusion impedance of $\mathrm{Li}$ ions and steady interfacial environment (Tables $\mathrm{S} 1$ and S2). Under harsher conditions of $2 \mathrm{~mA} \mathrm{~cm}^{-2}$ and $2 \mathrm{~mA} \mathrm{~h} \mathrm{~cm}^{-2}$, the CE for SMGCS cell could reach $98 \%$ and remains stable for 100 cycles. The cell of pristine $\mathrm{Cu}$ fails after 30 cycles, exhibiting a $\mathrm{CE}$ as low as $78 \%$ (Fig. 3d). The corresponding Li plating/stripping behavior is probed by the charging-discharging voltage profiles, as plotted in Fig. $3 e$ and $\mathrm{f}$. The charging voltage profiles of pristine $\mathrm{Cu}$ are apparently shorter than the discharging ones in the time scale, implying the large irreversible capacity loss. On the contrary, the Li loss of SMGCS cell is negligible because of its high CE. Moreover, the long-term voltage hysteresis between charging and discharging voltage plateau can be observed. The cell of SMGCS realizes a much smaller and quite stable hysteresis, while the polarization of pristine $\mathrm{Cu}$ is dramatically increased. A sharp and steep increase of hysteresis is observed in the 30th cycle, in complete agreement with the failure of $\mathrm{Li}$ anodes with the drastic increase in $\mathrm{Li}$-ion diffusion resistance [35]. The promoted SMGCS Li metal anode contributes largely to small polarization, high $\mathrm{Li}$ utilization and stable cycling. Furthermore, in order to achieve much higher capacities (i.e., can be much more deeply cycled), we analyzed the $\mathrm{Li} \| \mathrm{Cu}$ cells at high capacities of 5 and $10 \mathrm{~mA} \mathrm{~h} \mathrm{~cm}^{-2}$ (Fig. $3 \mathrm{~g}$ ). The cell of pristine $\mathrm{Cu}$ is not even cyclable, whereas stable CE $(\sim 97.8 \%)$ could still be obtained for SMGCS, further indicating its superior capability to render deeply cyclable Li metal anodes.

Usually, excess Li is required for practical use of LMBs to avoid fast capacity degradation. In this regard, we evaluated the $\mathrm{CE}$ of $\mathrm{Li}$ metal anodes with different amounts of excess Li (Fig. 4a). Notably, when $200 \%$ excess $\mathrm{Li}$ is used, the average CE of SMGCS Li metal anode could reach a high value of $98.51 \%$. The average CE gradually decreases when the amount of excess $\mathrm{Li}$ is reduced. Significantly, a high average CE of $98.15 \%$ still can be obtained when only $50 \%$ excess $\mathrm{Li}$ is applied. Under the same conditions, sharp peak occurs at charging process for pristine $\mathrm{Cu}$ and with little $\mathrm{LiNO}_{3}$ because active $\mathrm{Li}$ metal on $\mathrm{Cu}$ is quickly exhausted and the remaining $\mathrm{Li}$ could not be removed. Further estimations for the reversibility and stability of Li metal anode with $50 \%$ excess Li were also conducted. With $1.5 \mathrm{~mA} \mathrm{~h} \mathrm{~cm}^{-2} \mathrm{Li}$ deposited, the electrode was then stripped/deposited with capacity of $1.0 \mathrm{~mA} \mathrm{~h} \mathrm{~cm}{ }^{-2}$ until the excess Li was entirely consumed (Fig. $4 \mathrm{~b}$ and Fig. S10). The charge plateau remains stable for over $100 \mathrm{Li}$ stripping/plating cycles (or $200 \mathrm{~h}$ ), which is a satisfactory result when only $50 \%$ excess $\mathrm{Li}$ is applied. The terminal charge potential in each cycle is also demonstrated in Fig. 4c, which remains stable after more than 100 cycles. These results highlight the excellent stability of SMGCS Li metal and the possibility to realize application of Li metal full-cell with excellent cycling performance, high energy density and security. To further demonstrate the feasibility of such a structure in practical applications, predeposited $\mathrm{Li}$ anodes with slight excess $\mathrm{Li}$ were used to construct full cells (Fig. 4d). Because of its proven high operation voltage window $(4.45 \mathrm{~V})$, high practical capacity $\left(150 \mathrm{~mA} \mathrm{~h} \mathrm{~g}^{-1}\right)$ and compatibility with carbonate electrolytes, we select commercial $\mathrm{LiCoO}_{2}$ (LCO) as the cathode with a high mass loading $\sim 18.5 \mathrm{mg} \mathrm{cm}^{-2}$. The full cells paired with predeposited $\mathrm{Li}$ metal on pristine $\mathrm{Cu}$ exhibit a dramatic capacity fading and rather low CEs. In sharp contrast, the full cells of LCO $\mid 50 \%$ excess $\mathrm{Li}$ and even LCO $\mid 20 \%$ excess $\mathrm{Li}$ for SMGCS Li anode both deliver excellent cycling stability and significantly improved CEs, which are extraordinary results for Li-metal full cells (Fig. 4e, f and Figs S11 and S12). Furthermore, the full cells of LCO\| $20 \%$ excess $\mathrm{Li}$ 

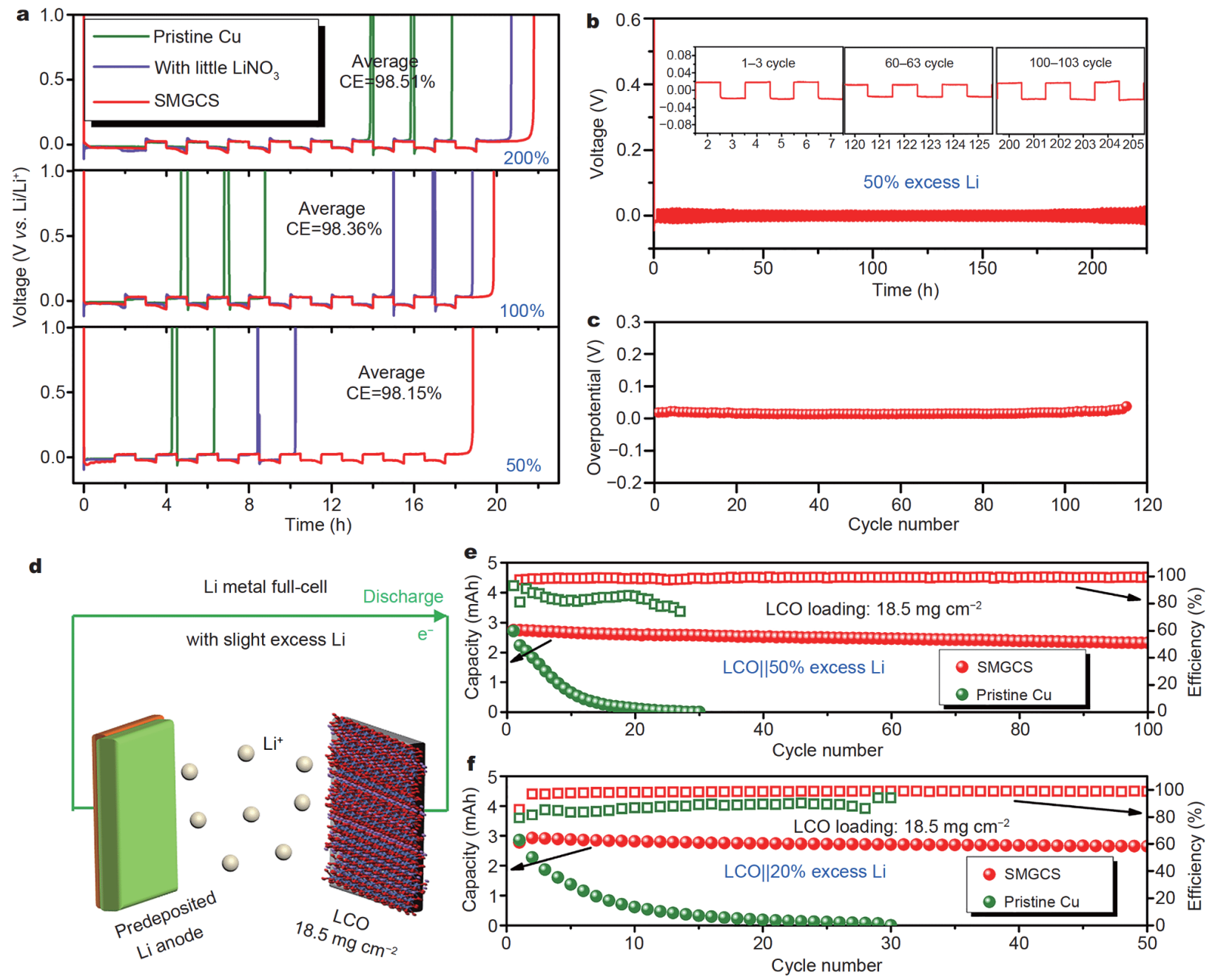

Figure 4 Highly reversible LMB with slight excess Li. (a) The CEs of Li anodes with different amounts of excess Li. (b) The long-term cycling performance of Li metal anode with $50 \%$ excess Li. (c) The terminal charge voltage in each cycle of (b). (d) The schematic structure of Li metal fullcell. $\mathrm{Cu}$ or SMGCS electrodes were pre-deposited with certain amounts of Li as anodes and were paired with LCO cathodes to constitute Li metal fullcells. The cycle lives and CEs of the Li metal full-cells of (e) LCO $\mid 50 \%$ excess Li and (f) LCO $\mid 20 \%$ excess Li. The mass loading of LCO cathode was about $18.5 \mathrm{mg} \mathrm{cm}^{-2}$.

(SMGCS) delivered much better stability than the full cell of $\mathrm{LCO} \| 200 \%$ excess $\mathrm{Li}$ (pristine $\mathrm{Cu}$ ), further indicating the superiority of SMGCS for safer large-scale practical application of $\mathrm{Li}$ metal anodes (Fig. S13). Moreover, coupled with the high average discharging voltage of $4 \mathrm{~V}$, the full cell of LCO $\mid 20 \%$ excess Li could afford a high specific energy of $572 \mathrm{~W} \mathrm{~h} \mathrm{~kg}^{-1}$ based on the total mass of electrode active materials. In addition, electrochemical performances of $\mathrm{Li} \| \mathrm{NCM}$ with pristine $\mathrm{Li}$ and $\mathrm{AgNO}_{3}$ modified $\mathrm{Li}$ with cathode areal loading of $20 \mathrm{mg} \mathrm{cm}^{-2}$ were also investigated. The $\mathrm{AgNO}_{3}$-modified $\mathrm{Li}$ demonstrated much better battery performance (Fig. S14). Therefore, the performance of SMGCS anode is superior to recently reported Li-metal anodes in terms of achieved area capacities and current densities. In addition, the high degree of Li utilization in full-cells demonstrates their potential for practical implementation in high safety-level and energy-density Li batteries (Table S3).

\section{CONCLUSIONS}

In summary, highly efficient utilization of SMGCS Li metal (merely $20 \%$ excess anodic capacity in Li metal fullcell) in carbonate electrolytes was realized by introducing $\mathrm{AgNO}_{3}$ membranes. Through a facile displacement reaction between $\mathrm{AgNO}_{3}$ and Li during Li plating, in-situ silver lithiophilic sites and nitrate-rich layers can be obtained on the surface of Li metal anode. The as-proposed design could act as an SMGCS towards guiding and 
confining Li deposition: Ag acts as lithiophilic nucleation sites to reduce Li nucleation overpotential and guide the $\mathrm{Li}$ initial nucleation; the nitrate-rich layer is helpful to confine and homogenize Li-ion flux, thus rendering reversible and dendrite-free $\mathrm{Li}$ metal deposition. Consequently, high CE of $~ 99 \%$ was achieved for over 300 cycles and Li metal can be deeply cycled at high capacity of $10 \mathrm{~mA} \mathrm{~h} \mathrm{~cm}{ }^{-2}$. More importantly, the Li metal full-cell with merely $20 \%$ excess Li (i.e., Li utilization of $83.3 \%$ ) demonstrated significantly improved reversibility and CEs with almost all of the cathode capacity delivered. The present study realized the goals of excellent cycling efficiency in commercial carbonate-based liquid electrolytes and highly efficient Li utilization in Li metal full-cell. This is expected to favor the safe practical use of Li metal anodes, opening a new horizon towards the future development of Li metal full-cells.

\section{Received 25 June 2020; accepted 17 August 2020; published online 3 November 2020}

1 Xu W, Wang J, Ding F, et al. Lithium metal anodes for rechargeable batteries. Energy Environ Sci, 2014, 7: 513-537

2 Lin D, Liu Y, Cui Y. Reviving the lithium metal anode for highenergy batteries. Nat Nanotech, 2017, 12: 194-206

3 Goodenough JB, Kim Y. Challenges for rechargeable Li batteries. Chem Mater, 2009, 22: 587-603

4 Ma J, Meng F, Yu Y, et al. Prevention of dendrite growth and volume expansion to give high-performance aprotic bimetallic Li$\mathrm{Na}$ alloy- $\mathrm{O}_{2}$ batteries. Nat Chem, 2019, 11: 64-70

5 Zhao CZ, Chen PY, Zhang R, et al. An ion redistributor for dendrite-free lithium metal anodes. Sci Adv, 2018, 4: eaat3446

6 Li G, Liu Z, Huang Q, et al. Stable metal battery anodes enabled by polyethylenimine sponge hosts by way of electrokinetic effects. Nat Energy, 2018, 3: 1076-1083

7 Albertus P, Babinec S, Litzelman S, et al. Status and challenges in enabling the lithium metal electrode for high-energy and low-cost rechargeable batteries. Nat Energy, 2018, 3: 16-21

8 Qiu F, Li X, Deng H, et al. A concentrated ternary-salts electrolyte for high reversible Li metal battery with slight excess Li. Adv Energy Mater, 2019, 9: 1803372

9 Liu Y, Lin D, Li Y, et al. Solubility-mediated sustained release enabling nitrate additive in carbonate electrolytes for stable lithium metal anode. Nat Commun, 2018, 9: 3656

10 Alvarado J, Schroeder MA, Pollard TP, et al. Bisalt ether electrolytes: a pathway towards lithium metal batteries with $\mathrm{Ni}$-rich cathodes. Energy Environ Sci, 2019, 12: 780-794

11 Qian J, Henderson WA, Xu W, et al. High rate and stable cycling of lithium metal anode. Nat Commun, 2015, 6: 6362

12 Yamada Y, Wang J, Ko S, et al. Advances and issues in developing salt-concentrated battery electrolytes. Nat Energy, 2019, 4: 269-280

13 Shi Q, Zhong Y, Wu M, et al. High-capacity rechargeable batteries based on deeply cyclable lithium metal anodes. Proc Natl Acad Sci USA, 2018, 115: 5676-5680

14 Zhang XQ, Cheng XB, Chen X, et al. Fluoroethylene carbonate additives to render uniform $\mathrm{Li}$ deposits in lithium metal batteries.
Adv Funct Mater, 2017, 27: 1605989

15 Markevich E, Salitra G, Chesneau F, et al. Very stable lithium metal stripping-plating at a high rate and high areal capacity in fluoroethylene carbonate-based organic electrolyte solution. ACS Energy Lett, 2017, 2: 1321-1326

16 Zhu B, Jin $\mathrm{Y}, \mathrm{Hu} \mathrm{X}$, et al. Poly(dimethylsiloxane) thin film as a stable interfacial layer for high-performance lithium-metal battery anodes. Adv Mater, 2017, 29: 1603755

17 Yan K, Lee HW, Gao T, et al. Ultrathin two-dimensional atomic crystals as stable interfacial layer for improvement of lithium metal anode. Nano Lett, 2014, 14: 6016-6022

18 Gao Y, Zhao Y, Li YC, et al. Interfacial chemistry regulation via a skin-grafting strategy enables high-performance lithium-metal batteries. J Am Chem Soc, 2017, 139: 15288-15291

19 Lu LL, Ge J, Yang JN, et al. Free-standing copper nanowire network current collector for improving lithium anode performance. Nano Lett, 2016, 16: 4431-4437

20 Jin C, Sheng O, Luo J, et al. 3D lithium metal embedded within lithiophilic porous matrix for stable lithium metal batteries. Nano Energy, 2017, 37: 177-186

21 Yan K, Lu Z, Lee HW, et al. Selective deposition and stable encapsulation of lithium through heterogeneous seeded growth. Nat Energy, 2016, 1: 16010

22 Liu W, Mi Y, Weng Z, et al. Functional metal-organic framework boosting lithium metal anode performance via chemical interactions. Chem Sci, 2017, 8: 4285-4291

23 Li W, Yao H, Yan K, et al. The synergetic effect of lithium polysulfide and lithium nitrate to prevent lithium dendrite growth. Nat Commun, 2015, 6: 7436

24 Li L, Jacobs R, Gao P, et al. Origins of large voltage hysteresis in high-energy-density metal fluoride lithium-ion battery conversion electrodes. J Am Chem Soc, 2016, 138: 2838-2848

25 Wang $\mathrm{F}, \mathrm{Yu} \mathrm{HC}$, Chen $\mathrm{MH}$, et al. Tracking lithium transport and electrochemical reactions in nanoparticles. Nat Commun, 2012, 3: 1201

26 Tu Z, Choudhury S, Zachman MJ, et al. Fast ion transport at solidsolid interfaces in hybrid battery anodes. Nat Energy, 2018, 3: 310316

27 Yan C, Cheng XB, Yao YX, et al. An armored mixed conductor interphase on a dendrite-free lithium-metal anode. Adv Mater, 2018, 30: 1804461

28 Choudhury S, Tu Z, Stalin S, et al. Electroless formation of hybrid lithium anodes for fast interfacial ion transport. Angew Chem Int Ed, 2017, 56: 13070-13077

29 Canongia Lopes JN, Deschamps J, Pádua AAH. Modeling ionic liquids using a systematic all-atom force field. J Phys Chem B, 2004, 108: 2038-2047

30 Canongia Lopes JN, Pádua AAH. Molecular force field for ionic liquids composed of triflate or bistriflylimide anions. J Phys Chem B, 2004, 108: 16893-16898

31 Soetens JC, Millot C, Maigret B. Molecular dynamics simulation of $\mathrm{Li}^{+} \mathrm{BF}_{4}^{-}$in ethylene carbonate, propylene carbonate, and dimethyl carbonate solvents. J Phys Chem A, 1998, 102: 1055-1061

32 Zhang R, Chen XR, Chen X, et al. Lithiophilic sites in doped graphene guide uniform lithium nucleation for dendrite-free lithium metal anodes. Angew Chem Int Ed, 2017, 56: 7764-7768

33 Yang CP, Yin YX, Zhang SF, et al. Accommodating lithium into 3D current collectors with a submicron skeleton towards long-life lithium metal anodes. Nat Commun, 2015, 6: 8058

34 Li P, Lan H, Yan L, et al. Lithiation/delithiation behavior of silver 
nitrate as lithium storage material for lithium ion batteries. ACS Sustain Chem Eng, 2017, 5: 5686-5693

35 Cheng XB, Yan C, Peng HJ, et al. Sulfurized solid electrolyte interphases with a rapid $\mathrm{Li}^{+}$diffusion on dendrite-free $\mathrm{Li}$ metal anodes. Energy Storage Mater, 2018, 10: 199-205

Acknowledgements We acknowledge support from the National Natural Science Foundation of China (51622208, 21703149, and 51872193) and the Priority Academic Program Development of Jiangsu Higher Education Institutions (PAPD).

Author contributions Zhou J, Zhou X, Qian T and Yan C conceived the idea and designed the experiment. Zhou J, Lu H, Ji H and Sun Y carried out the synthesis, characterization, electrochemical experiments and calculation. All authors participated in the data analysis and contributed to the manuscript writing.

Conflict of interest There are no conflicts to declare.

Supplementary information Supporting data are available in the online version of the paper.

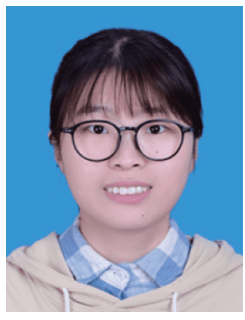

Jinqiu Zhou is a $\mathrm{PhD}$ student under the supervision of Prof. Chenglin Yan at Soochow University. Her research interests focus on lithium battery.

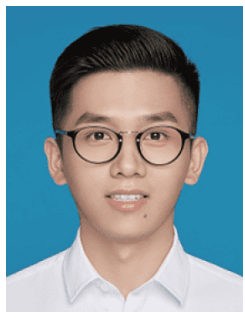

Haoliang Lu studies as a master under the supervision of Prof. Chenglin Yan at Soochow University. His research interests focus on zincion batteries, graphene energy storage and its applications.

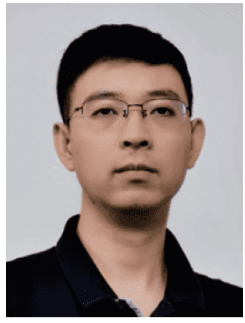

Xi Zhou received his PhD degree from Nanjing University in 2016. Hs is now a Research Associate at the Institute of Chemical Industry of Forestry Products in Chinese Academy of Forestry. His research interests focus on biomassbased functional materials and their applications in energy storage, photoelectric sensor and biosorption.

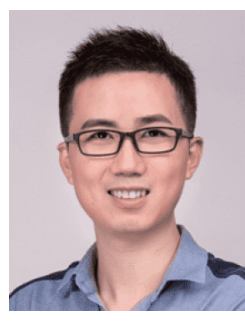

Tao Qian received his $\mathrm{PhD}$ degree from Nanjing University in 2014. Hs is now a associate researcher at Soochow University. His research interests focus on energy conversion/storage including lithium battery and electrochemical catalysis.

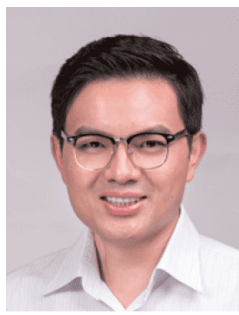

Chenglin Yan joined Soochow University as a distinguished professor in 2014. He is now the Dean of the College of Energy. His research is totally related to novel design of lithium battery, electrochemical catalysts and their applications in energy storage and conversion.

\section{模拟导弹制导约束系统实现锂全电池中锂金属的 高效利用}

周金秋 ${ }^{1 \dagger}$, 陆豪量 ${ }^{1 \dagger}$, 周希 ${ }^{2 *}$, 季浩卿 ${ }^{1}$, 孙雅雯 ${ }^{1}$, 钱涛 $^{1^{*}}$, 晏成林 ${ }^{*}$

摘要 金属锂的有效利用是实现锂金属全电池高安全性和高能量 密度应用的关键. 本文中, 我们报道了一种仅含 $20 \%$ 过量锂且可逆 性和库仑效率显着提高的锂金属全电池. 我们通过设计巧妙的模 拟导弹制导约束系统(SMGCS), 构建兼容的亲锂银位点和硝酸盐 层来引导和限制锂的沉积. 银位点充当有效的锂沉积位点, 吸引锂 离子, 引导锂的初始成核. 生成的硝酸盐层提供了有利于均匀限域 且高容量锂沉积的界面环境, 这在理论上已通过分子动力学 (MD) 模拟得到了验证. 这两种优点相结合, 实现了坚固且无枝晶的锂沉 积以及含有极少量过量锂的锂金属全电池的应用. 并且该锂金属 在碳酸酯电解液中表现出优异的循环效率(300次以上的循环中库 伦效率约 $99 \%$ ), 还可以在 $10 \mathrm{~mA} \mathrm{~h} \mathrm{~cm}^{-2}$ 的高容量下进行深度循环. 本文中锂金属前所未有的高利用率为未来高效锂金属全电池的发 展开辟了一条新的道路. 